\title{
Nutzungskonflikte bewältigen
}

\author{
Wie kann die ökologische Funktionsfähigkeit der Stromlandschaft Elbe erhalten \\ und verbessert werden? Seit 1996 fördert das Bundesministerium für Bildung \\ und Forschung die Entwicklung entsprechender Nutzungs- und Entwicklungskon- \\ zepte im Rahmen des Forschungsverbunds Elbe-Ökologie.
}

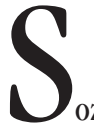

Von Alexandra Dehnhardt und Jürgen Meyerhoff neben naturwissenschafticher Schwerpunkt des interdisziplinär ausgerichteten Programms. Ihnen kommt vor allem die Aufgabe $\mathrm{zu}$, Grundlagen für die Lösung zu erwartender Nutzungskonflikte zu schaffen und so einen wichtigen Beitrag zur umweltpolitischen Zielfindung zu leisten (1). An der Elbe könnten durch Deichrïckverlegungen insgesamt rund 15.000 Hektar Retentionsflächen wieder gewonnen und ökologisch wertvolle Auenwälder reaktiviert werden. Die Erreichung des ökologischen Zieles führt allerdings zu Konflikten mit der bisherigen Flächennutzung. Um sie einer „Lösung“ zuführen zu können, sind Informationen erstens über Art und institutionelle Rahmenbedingungen des Nutzungskonfliktes sowie zweitens über den gesellschaftlichen Wert der verschiedenen Nutzungen erforderlich.

\section{Nutzungskonflikte aus Deichrückverlegungen}

Deichrückverlegungen führen zur Verdrängung bisheriger Landnutzungsformen und damit zu Nutzungskonflikten. Deren Identifikation und Bewertung sowie die Ableitung von Maßnahmenkonzepten bilden den Schwerpunkt der sozioökonomischen Forschung des Instituts für ökologische Wirtschaftsforschung (IÖW) im Verbundprojekt Rückgewinnung von Retentionsflächen und Altauenreaktivierung an der Mittleren Elbe in Sachsen-Anhalt.

Grundlage ist eine Konfliktanalyse, die Gewinner und Verlierer einer Deichrïckverlegung aufzeigt. Klassische Vertreterin eines in der Regel stark konfliktorientierten Verhältnisses zu den Anforderungen des Naturschutzes ist die Landwirtschaft als dominierende Flächennutzerin. Das Konfliktpotenzial und in Abhängigkeit davon die Umsetzungsbedingungen hängen hier stark von Kompensationsmöglichkeiten und der regional spezifischen Bedeutung der Landwirtschaft ab.
Vergleichsweise günstige Umsetzungsbedingungen ergeben sich im Projektgebiet Sandau, wo stärkere Rückzugstendenzen der Landwirtschaft - etwa durch extensivere Produktion oder die Orientierung an Landschaftspflegeleistungen - erkennbar sind. Es ist davon auszugehen, dass sich diese Tendenz im Hinblick auf die künftige agrarpolitische Entwicklung verstärken wird und daher die Nutzungskonflikte abnehmen. Eine Anpassung der Kompensationen an regionale Landnutzungsschwerpunkte - zum Beispiel eine theoretisch denkbare Quotenerhöhung für Mutterkühe als Form auenangepasster Landwirtschaft - würde die mit der Projektrealisierung verbundenen Kosten in diesem Bereich insgesamt senken und damit Konfliktlösungsmöglichkeiten eröffnen.

Direkte Nutzen einer Deichrückverlegung sind demgegenüber für die Fischbestände durch Verbesserung der Laichbedingungen, aber auch für Teile der Forstwirtschaft zu erwarten. Die Wiederherstellung einer natürlichen Überflutungsdynamik in Altauenwäldern würde vermutlich zu einer Steigerung ihrer Produktivität und damit der Holzerträge führen.

\section{Ökonomische Nutzenermittlung}

Mit einer genaueren Ermittlung und Gewichtung der Nutzen beschäftigt sich das Projekt Monetäre Bewertung einer nachbaltigen Entwicklung der Stromlandschaft Elbe. Dieses wird gemeinsam vom IÖW und der Technischen Universität Berlin bearbeitet. Darüber hinaus soll ein Beitrag zur Entwicklung der ökonomischen Bewertungsmethoden geleistet werden. Verfolgt werden zwei Bewertungsansätze:

- Erstens die Ermittlung der direkten Zahlungsbereitschaft mit Hilfe der Contingent Valuation (CV). Bisherige Untersuchungen haben gezeigt, dass nicht-nutzungsabhängige Wertschätzungen einen bedeutenden Teil des ökonomischen Wertes natïrlicher Ressourcen ausmachen können (2). Nur durch die Etablierung eines hypothetischen Marktes mit Hilfe von Interviews im Rah- men einer CV lassen sich auch diese Wertschätzungen ermitteln. Durch ein Befragungsinstitut sollen daher nicht nur Personen im Elbeeinzugsgebiet, sondern auch in zwei anderen Regionen der Bundesrepublik befragt werden. Ein weiterer Schwerpunkt liegt darauf, durch die Hinzuziehung von Erkenntnissen der Sozial-Psychologie die Ergebnisse besser zu erklären.

- Weiterhin sollen exemplarisch ökologische Leistungen von Flussauen mit Hilfe „objektiver“ Bewertungsverfahren bewertet werden. Überschwemmungsauen übernehmen zum Beispiel eine entsorgende Funktion als Nährstoffsenke und Schadstofffilter. Die objektiven Verfahren zeichnen sich dadurch aus, dass sie formal Ursache-Wirkungs-Beziehungen zur Grundlage haben. Ansatzpunkt für die Bewertung sind hier in der Regel umweltpolitische Ziele. Gemessen wird dann der Beitrag der ökologischen Leistung zur Erreichung des jeweiligen Umweltzieles. Als Verfahren sind zu nennen: Ausgaben für präventive Maßnahmen, Bewertung von Veränderungen der Produktivität, Wiederherstellungs- und Schadensvermeidungskosten.

Die Ergebnisse der CV und der indirekten Verfahren liefern jeweils einen Baustein für den gesamten ökonomischen Wert einer nachhaltigen Entwicklung in der Stromlandschaft Elbe. Dieser Wert soll dann für Kosten-Nutzen-Analysen von Einzelmaßnahmen wie etwa Deichrückverlegungen herangezogen werden. Die Ergebnisse des Vorhabens werden auf einem Workshop im Frühjahr 2002 vorgestellt.

\section{Anmerkungen}

(1) Vgl. für einen Überblick Bornhöft, Achim/ Meyerhoff, Jürgen: Der Beitrag der Umwelt- und Sozioökonomie für die ökologische Forschung in der Stromlandschaft Elbe. IÖW-Schriftenreihe 126/97, Berlin 1997.

(2) Siehe Meyerhoff, Jürgen: Ökonomische Bewertung ökologischer Leistungen. Stand der Diskussion und mögliche Bedeutung für die Elbe-Ökologie. IÖW-SR 136/98, Berlin 1998.

\section{Die Autorlnnen}

Alexandra Dehnhardt ist wissenschaftliche Mitarbeiterin im Forschungsfeld Umweltökonomie und -politik des IÖW, Jürgen Meyerhoff ist wissenschaftlicher Mitarbeiter am Institut für Management in der Umweltplanung der TU Berlin.

Kontakt: IÖW, Potsdamer Str. 105, 10785 Berlin. Tel. 030/884594-0, Fax 030/ 8825439, E-mail: Alexandra.Dehnhardt@ioew.de, meyerhoff@imup.tu-berlin.de 
(c) 20I0 Authors; licensee IÖW and oekom verlag. This is an article distributed under the terms of the Creative Commons Attribution Non-Commercial No Derivates License (http://creativecommons.org/licenses/by-nc-nd/3.o/), which permits unrestricted use, distribution, and reproduction in any medium, provided the original work is properly cited. 